\title{
El Tribunal Constitucional en Polonia y la interpretación de normas jurídicas *
}

The constitutional court in Poland and the interpretation of legal norms

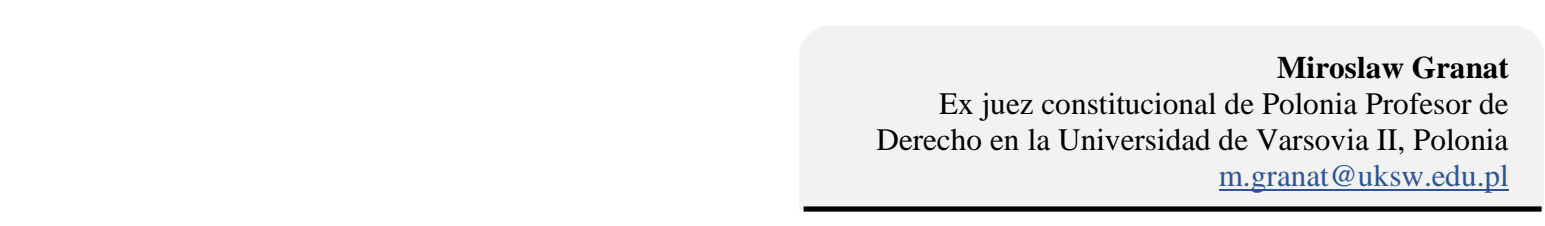

Recibido: 25/09/2019 Aprobado: 01/11/2019

DOI: $10.25054 / 16576799.2805$

\section{RESUMEN}

El artículo desarrolla las funciones de control constitucional y de interpretación de las normas por parte del Tribunal Constitucional de Polonia. En el texto se desarrollan los fundamentos teóricos de la interpretación que realiza el juez constitucional polaco, quien juzga las normas y las interpreta a la luz de la Constitución de 1997. Dentro de este desarrollo que ha venido realizando el Tribunal, se ejemplifican varios casos que ponen de manifiesto el gran poder de los jueces en Polonia, lo que le ha valido el apelativo de "juezocracia". Finalmente se desarrolla unas características del sistema de control judicial constitucional, estableciendo su alcance.

\section{PALABRAS CLAVE}

Tribunal Constitucional en Polonia; Control Constitucional; Interpretación Pro-constitucional; Facultades del Juez Constitucional.

\begin{abstract}
The article develops the functions of constitutional control and interpretation of the norms by the Polish Constitutional Court. The text elaborates on the theoretical basis of the interpretation made by the Polish constitutional judge, who judges the rules and interprets them in the light of the 1997 Constitution. As part of the development of the Court, a number of cases are exemplified which highlight the great power of judges in Poland which has given it the title of "judge". Finally, the characteristics of the constitutional judicial control system are developed, establishing its scope.
\end{abstract}

\section{KEYWORDS}

Constitutional Court in Poland; Constitutional Control; Pro-constitutional Interpretation; Powers of the Constitutional Judge. 


\section{INTRODUCCIÒN}

En la doctrina del derecho polaco no existe ninguna teoría de la interpretación privilegiada que vincule la interpretación de la constitución o de cualquier otro texto legal. Por su parte, la jurisprudencia constitucional se ha dotado de algunos métodos y técnicas de interpretación de la constitución; algunas de ellas merecen ser calificadas como "teorías de la interpretación".

Lo primero que hay que precisar es que es una teoría de la interpretación pro-constitucional de las normas. Se trata de interpretar las normas jurídicas vigentes para garantizar su conformidad con la constitución. Según la teoría de la interpretación pro-constitucional, las normas de rango inferior pueden tener significados diversos. Sin embargo, se supone que el juez constitucional (o cualquier otro juez) debe elegir un significado que sea el más fiel a la constitución. La interpretación proconstitucional es, por lo tanto, una de las teorías básicas y uno de los instrumentos comunes utilizados

\section{FUNDAMENTOS TEÓRICOS DE LA INTERPRETACIÓN DE LAS NORMAS POR EL JUEZ CONSTITUCIONAL}

El Tribunal Constitucional Polaco, como órgano encargado de juzgar las normas del derecho, es un órgano pasivo, distante y reservado. Su función primaria es "constitucionalizar" una norma sometida a examen. Solo en el momento en que el esfuerzo de interpretación fracasa puede producirse la excepción de una norma equivocada (es una solución extrema). La interpretación proconstitucional se autolimita en la medida en que no puede conducir a la creación de una norma nueva, ausente previamente del sistema jurídico.

El deber de buscar una interpretación proconstitucional radica en que las normas constitucionales tienen una fuerza suprema de derecho. La Constitución de la República de Polonia de 1997 expresamente habla del principio de constitucionalismo (art. 8, párr. 1), el cual significa que sus disposiciones representan el derecho supremo en Polonia y que deben aplicarse directamente, salvo que se disponga otra cosa en la Constitución (art. 8, párr. 2). Por lo tanto, es la base para la interpretación pro-constitucional de las normas.

En segundo lugar, se trata de un enfoque que tiene por objeto conceder un alcance directo a las disposiciones constitucionales. En efecto, se considera que algunas disposiciones constitucionales son tan concretas que se prestan a una aplicación inmediata. No necesitan una interpretación específica.

Por ejemplo: el párrafo 2 del artículo 98 de la Constitución dispone que las elecciones al $\operatorname{Sejm}^{l}$ y al Senado serán ordenadas por el presidente de la República. Éste fija su fecha en "un día feriado". El Tribunal Constitucional tuvo que examinar la conformidad de una disposición del Código Electoral con esa referencia constitucional. La disposición en cuestión, dejó la posibilidad de celebrar las elecciones presidenciales en dos días, el sábado y el domingo. El Tribunal Constitucional, en su decisión de 20 de julio de 2011 (expediente K9/11)2 , afirmó que el párrafo 2 del artículo $98 \mathrm{de}$ la Constitución sólo se refería al domingo (por lo tanto, las elecciones solo podían celebrarse un día).

En tercer lugar, existe una teoría de la interpretación derivada. Según esta teoría, la norma se deriva de la disposición. A veces se dice que no puede haber una norma sin una interpretación previa. Además, el examen de la constitucionalidad se lleva a cabo a nivel de la interpretación de las disposiciones y no a nivel de las normas. Si se mantiene esta teoría, se rechaza la idea del impacto directo de las disposiciones constitucionales. Todas las normas deben derivarse de las disposiciones. En consecuencia, incluso una disposición simple como el párrafo 1 del artículo 98, que dispone que las elecciones al Sejm y al Senado son ordenadas por el presidente de la República "un día festivo", exige que se detecte una norma. Según este enfoque, el término "feriado" puede referirse tanto al sábado como al domingo. 
Digamos como una nota al margen, que de conformidad con el párrafo 2 del artículo 98 de la Constitución, el Código Electoral de 2011 prevé elecciones de dos días de duración. A raíz de la citada decisión del tribunal constitucional, esta disposición del Código Electoral ha sido objeto de revisión. Por lo tanto, se precisa que las elecciones se celebran en un día y es el domingo. Respecto a esto, cabe señalar que algunos jueces que habían formado parte del cuerpo jurisdiccional, que se ocupaba de esta cuestión, presentaron opiniones discrepantes a esta decisión. Alegaron que no era posible descubrir el contenido de la norma del párrafo 2 del artículo 98 sin recurrir a la interpretación derivada. Y, siguiendo esta interpretación, se sentían capaces de decir que el sábado era también un "día festivo"3.

Lejos de decidir cuál de las dos teorías de interpretación de las disposiciones constitucionales es la más adecuada, se debe precisar que los dos enfoques, directos y derivados, marcan los extremos en la interpretación de la Constitución. En Polonia, los políticos están debatiendo recientemente la aprobación de una nueva Constitución que sustituiría a la de 1997 y que sería clara desde el primer momento, para acabar con la "juezocracia" que se ha apropiado del poder bajo el ojo protector de la Constitución en vigor. Esta famosa "juezocracia" sigue interpretando la Constitución, entre otras cosas, mediante una interpretación derivada. Dicho esto, la idea de adoptar una Constitución "perfectamente clara", es decir, sin interpretaciones, por razones evidentes, es una utopía pura.

En mi opinión, los fundamentos teóricos de la interpretación por parte del juez constitucional están relacionados, en primer lugar, con un marco normativo de la Constitución de 1997. Tras la caída del régimen comunista, la Constitución polaca tuvo una génesis consensuada, es decir fruto de acuerdos. Muchas disposiciones constitucionales son deliberadamente vagas. Además, se puso un Preámbulo. De ello se deduce la dirección que debe adoptarse para interpretar las demás disposiciones constitucionales.

Las disposiciones constitucionales fundamentales están establecidas en el principio del derecho.
Según la doctrina polaca, los principios del derecho son normas de orden universal, por una parte, y de gran densidad jurídica, por otra. Su fundamento axiológico es especialmente marcado, ya que apelan a los valores. Sin detenerse en las grandes controversias doctrinales relativas a la percepción de los principios del derecho constitucional (lo cual es una materia muy compleja), no cabe duda que la aplicación por parte del Tribunal Constitucional de estas normas -principios del derecho- requiere, un esfuerzo de interpretación previo. Normalmente solo contienen un "extracto" de la solución deseada por el otorgante. Esto tiende a dar al legislador ordinario, un direccionamiento sobre el objeto a alcanzar. Considérese, por ejemplo, el principio de la libertad humana, que fue objeto de la interpretación del tribunal constitucional. El Tribunal adoptó la decisión K 6/14 de 2016 relativa a un supuesto "hombre - monstruo", llamado así por haber cometido múltiples crímenes deleznables.

Hay que precisar que, de conformidad con el párrafo 1 del artículo 31 de la Constitución, "La libertad del hombre está legalmente protegida". La cuestión es saber si las personas que han cometido los crímenes más abominables y han cumplido la pena de 25 años de prisión, pero cuya liberación podría poner en peligro la libertad de los demás, también pueden disfrutar de esa libertad. Entonces, ¿se podía aislar a esas personas de por vida colocándolas en instituciones especializadas para proteger a los demás? El Tribunal Constitucional afirmó en su decisión K 6/14, del 23 de noviembre de 2016, que dicho procedimiento, supervisado por un Tribunal competente, era admisible.

Hasta el 2015, el Tribunal pudo elaborar una serie de instrumentos para hacer frente a las colisiones de los principios del derecho consagrados en la Constitución. El ejemplo arriba citado ilustra una colisión entre el principio de libertad humana y el principio de seguridad de las demás personas. Los instrumentos aquí mencionados son, en efecto, directrices de interpretación (denominada interpretación en segundo grado). Este aspecto se desarrollará más adelante.

3 Las opiniones disidentes expresadas en la decisión K 9/11 se referían precisamente a esta cuestión. 
Varias disposiciones constitucionales cumplen la función de disposiciones marco (también pueden calificarse como disposiciones programáticas). Esto significa, en esencia, que necesitan ser materializadas por un legislador ordinario. La Constitución muestra lagunas que pueden colmarse con leyes ordinarias. Por ejemplo, la Constitución de 1997 establece derechos civiles de carácter socioeconómico. El párrafo 1 del artículo 67 garantiza a los ciudadanos un "derecho a la seguridad social en caso de incapacidad laboral debida a enfermedad, discapacidad o después de haber alcanzado la edad de jubilación. La extensión y las formas de seguridad social están previstas por la ley". La lectura que hace el legislador ordinario de esta disposición es bastante variada.

En efecto, durante un año civil, las prestaciones sociales de retiro y pensión de vejez están sometidas a una valoración con el mismo valor, lo que significa que las prestaciones en cuestión son aumentadas en una misma suma de dinero para todos. En el siguiente año, la valoración de las pensiones de retiro y vejez se realiza en porcentaje, siendo el porcentaje más elevado para los jubilados y pensionados más pobres y menos elevado para los que son relativamente ricos.

En cada caso, el legislador desglosa de forma diferente el alcance y las formas de seguridad social prevista por la Constitución. Las disposiciones legales en materia han sido recurridas reiteradamente ante el Tribunal Constitucional por infringir la disposición constitucional del artículo 67, apartado 1. El Tribunal ha sido entonces llevado a interpretar la disposición indicando lo que el derecho a la seguridad social implicaba y, sobre esa base, ha decidido sobre la constitucionalidad o inconstitucionalidad de las disposiciones legales.

En la Constitución polaca hay unas 80 disposiciones-delegaciones, es decir, disposiciones constitucionales que remiten a leyes ordinarias. Ello demuestra la riqueza de los problemas de interpretación que pueden surgir cuando se cruzan la Constitución y las leyes que la aplican.

No es difícil invocar aquí otros tipos de normas constitucionales que requieren una gran labor de interpretación debido a su especificidad. La Constitución contiene, por ejemplo, disposiciones que pertenecen a la categoría de cláusulas generales. Con el fin de comprenderlas, no basta con un sistema jurídico, sino que hay que buscar en otros lugares. Al referirse a un Estado democrático de derecho (art. 2 de la Constitución), algunos exegetas recurren a un modelo de Estado de Rechtsstaat (y en ese momento hacen hincapié en las características formales de la ley adoptada); otros relacionan el término Estado democrático de derecho con el rule of law (Estado de Derecho), considerando que el derecho supone contenidos concretos.

El mismo texto de la Constitución se caracteriza por la autonomía de los términos que emplea y ello no lo desliga de las consecuencias relacionadas con el problema de la interpretación en su integridad. Las nociones que aparecen en la Constitución no significan necesariamente lo mismo que en otras ramas del derecho y mucho menos en el lenguaje corriente. Tomemos el ejemplo de la "causa" del párrafo $1^{\circ}$ del artículo 45 de la Constitución polaca $^{4}$. Esta norma no se debe explicar con una relación con el Código Civil o el Código Penal.

Los conceptos que se desprenden de los actos jurídicos de rango inferior no determinan lo que contiene la Constitución. Cuanto más autónomo parece el término, como "libertad personal" o "bien común", más necesaria es su interpretación. La Constitución habla su propio idioma, distinto de los utilizados en otras ramas del derecho (como por ejemplo las disposiciones detalladas del Código Penal), lo cual pone de relieve los problemas de interpretación de la Constitución.

El problema de la interpretación de la Constitución adquiere cada vez más importancia. Esto se debe a que algunas teorías borran las diferencias entre la Constitución y su interpretación. No es de extrañar que las controversias sobre la interpretación se asimilen a veces a la teoría de la Constitución en un enfoque adoptado por Bockenforde (2000), o bien que la interpretación de la Constitución, entre en el campo de la identidad de la Constitución. La materia de interpretación abarca varios conceptos de la filosofía del derecho y tiene el deber de crear instrumentos (marcos) para interpretar la Carta Política.

4 Este artículo dice literalmente: "Artículo 45. Todos tienen el derecho a un juicio justo y público, sin dilaciones indebidas, ante un Tribunal competente, imparcial e independiente". (Nota del traductor) 
En resumen, el carácter de las disposiciones constitucionales dificulta su lectura literal, favoreciendo, por el contrario, el trabajo de interpretación y el redescubrimiento. $\mathrm{La}$ interpretación de la Constitución se hace, pues, naturalmente. La interpretación no es un complemento de la Constitución. Las disposiciones constitucionales desprovistas de interpretación serían probablemente inútiles. El ejercicio de la interpretación de la Carta Política es de una importancia fundamental para su aplicación de forma efectiva, pues ella permite a las normas adquirir un contenido particular.

\section{MÉTODOS Y TÉCNICAS DE INTERPRETACIÓN DE LAS NORMAS POR EL JUEZ CONSTITUCIONAL}

Con el fin de interpretar las disposiciones constitucionales, así como otros textos legales, aplicamos diferentes métodos de interpretación en función del problema que nos proponemos resolver. Son los métodos universalmente conocidos de la ciencia jurídica y en particular una interpretación lingüística, una interpretación lógica, una interpretación funcional y, por último, una interpretación teleológica. Estos temas no se desarrollarán en este punto pues se considera que sus nombres revelan su esencia profunda.

No obstante, si existen varios enfoques de interpretación posibles de una determinada disposición, la aplicación de los métodos mencionados debe ir precedida de la adopción de las directivas que pongan un poco de orden a su uso. Se trata esencialmente de establecer una jerarquía de aplicación de estos métodos. Por lo general, estas directivas se consideran como interpretación de segundo grado, ya que determinan la primacía de uno de los métodos tradicionales de la interpretación.

Una interpretación dinámica resulta importante en la interpretación de la Constitución por parte del juez constitucional. Este tipo de interpretación se refiere a la interpretación funcional, sistémica o teleológica. Por otro lado, una interpretación estática, basada en un enfoque lingüístico, claramente tiene menos peso.

La interpretación pro-constitucional de las normas jurídicas vigentes, mencionada en la parte preliminar, es una variante de la interpretación dinámica. Su objetivo es dar a la disposición objeto de examen, un significado acorde con la constitución. El juez del tribunal constitucional utiliza una amplia gama de métodos de interpretación para garantizar la convergencia de la disposición y la constitución. La teoría del impacto directo de las disposiciones constitucionales se asemeja a la interpretación estática.

Como se acaba de exponer, el principio de constitucionalismo requiere que el juez busque una interpretación pro-constitucional. En la práctica habitual, esto se traduce en decisiones sobre la interpretación y sobre el alcance de la norma superior. Estos dos son nuevos instrumentos que permiten tomar decisiones basándose principalmente en la interpretación derivada. Dicho en otras palabras, son instrumentos suprakelseniano que ayudan a reglar ciertos aspectos.

Hasta los años noventa del siglo XX, las decisiones del tribunal constitucional se dividían en dos categorías. Se trataba, entre otras cosas, de decisiones positivas (que confirmaban la constitucionalidad de una disposición objeto de examen) y decisiones negativas (que denegaban la constitucionalidad de una disposición objeto de examen).

Ambas categorías (positivas y negativas) se consideran como regla general decisiones sencillas. Por un lado, este par de decisiones positivasnegativas son muy kelsenianas. Por otro lado, han demostrado ser demasiado rudimentarias para expresar bien la especificidad del proceso de decidir sobre la constitucionalidad de las normas. En una decisión sobre la interpretación, el Tribunal Constitucional decide sobre la constitucionalidad de una disposición legal examinada, escoge un significado -entre los que ya se aplican en la práctica- que converge hacia una disposición constitucional (hacia un modelo o una referencia de control). Al hacerlo, el Tribunal reconoce la constitucionalidad (o inconstitucionalidad) de una disposición, bajo la condición que ella sea tomada en el sentido predeterminado.

El Tribunal lo expone en primer lugar en el dispositivo de su decisión y luego decide su constitucionalidad. Tal decisión no implica 
necesariamente la derogación de toda la disposición, sino que solo invalida el sentido contrario a la Constitución.

En cuanto a la decisión sobre el alcance, el Tribunal Constitucional establece y expone en la parte dispositiva, un sentido para la disposición examinada. Este sentido es conforme (o contrario) a la Constitución en las condiciones y en el marco determinados. Acá un ejemplo: una disposición del Código de la Carretera que permite a la policía retirar el permiso de conducción al conductor por un rebasamiento de la velocidad máxima autorizada de 50 kilómetros por hora es conforme a la Constitución. Sin embargo, esta misma disposición no prevé el rebasamiento de la velocidad máxima permitida en caso de fuerza mayor (como el transporte a un hospital de una persona gravemente enferma). En este sentido, el alcance de la disposición es inconstitucional ${ }^{5}$. Es razonable considerar que el Tribunal Constitucional disecciona la norma -por así decirlo-, con el objetivo de definir su ámbito de aplicación y decidir sobre su constitucionalidad.

Cabe señalar que estas nuevas categorías de decisiones adoptadas por el tribunal constitucional suelen suscitar críticas. En este contexto se pide un "gobierno de jueces". Es cierto que una cura aplicada a las disposiciones legales por el tribunal constitucional, que intenta demostrar su convergencia con la constitución a través de la interpretación, contiene una parte del restablecimiento del derecho. Sin embargo, es útil señalar que las críticas no provienen necesariamente de los círculos políticos, sino de los círculos jurídicos. El Tribunal Supremo, en particular, se opone a que el tribunal esté incumpliendo la legislación. En la práctica, en ocasiones el Tribunal Supremo denegaba a la persona afectada la reanudación del procedimiento (civil o penal) en el que debía dictarse una decisión del tribunal constitucional. Fue una forma indirecta de manifestar su desaprobación de las decisiones sobre la interpretación. Por ello, el Tribunal Constitucional trata de hacer el menor uso posible de estos nuevos instrumentos, utilizándolos únicamente como último recurso.

El papel y el peso de la interpretación varían en función del poder ejercido por el Tribunal
Constitucional. La interpretación de las disposiciones constitucionales y de otras normas del derecho tiene una importancia fundamental cuando se trata de un control de la constitucionalidad de las normas que el Tribunal examina debidamente (control por solicitud). En Polonia tenemos un control previo (preventivo) y un control consecutivo.

Por regla general, las decisiones más importantes del Tribunal Constitucional provenían de esos controles. Cuando es necesario realizar un control concreto, en particular con relación a un asunto ligado a una cuestión jurídica o una reclamación constitucional, la importancia de la interpretación se reduce. Por ejemplo, el Tribunal Constitucional se había pronunciado sobre la interpretación de la disposición relativa al derecho de toda persona a ser oída por un tribunal competente (artículo 45, párrafo 1 de la Constitución). En su notoria decisión sobre el examen del bachillerato, el Tribunal sostuvo que este derecho al "tribunal competente" está al servicio de todos, pero no en toda "causa". Por ello, según el Tribunal Constitucional, los tribunales administrativos no están en condiciones de examinar la regularidad de las pruebas del bachillerato en química ${ }^{6}$.

\section{FUNCIONES DE LA INTERPRETACIÓN DE LAS NORMAS SOBRE LAS FACULTADES DEL JUEZ CONSTITUCIONAL}

En Polonia, el papel clave en la interpretación de las disposiciones constitucionales corresponde al Tribunal Constitucional. El Tribunal define un significado de las disposiciones constitucionales y legales durante el ejercicio de sus funciones. Sin embargo, no tiene un poder independiente para interpretar la Constitución, como ocurre en algunos países, como Eslovaquia y Hungría.

Además, el hecho de que el Tribunal Constitucional haya interpretado la Constitución desde 2015 se ha convertido en objeto de críticas por parte de muchos políticos. Diversas enmiendas a la ley, formuladas por el Tribunal constitucional (denominadas leyes de corrección), han conducido a una marginación de la jurisdicción constitucional.

5 Sentencia del Tribunal Constitucional K 24/15, del 11 de octubre de 2016, OTK ZU 2016A, apartado 77

6 Véase: sentencia del Tribunal Constitucional SK 29/13, OTK ZU 2015, nº 6A, apartado 83. 
Es preciso recordar así que hasta 1997 (año de la adopción de una nueva Constitución), el Tribunal Constitucional tenía una competencia específica: dar "una interpretación universalmente vinculada de las leyes". Era un medio para asegurarse de que había una interpretación "oficial" de una disposición de la ley ordinaria marcada de equívoca. Así pues, el Tribunal Constitucional procedía a la interpretación y la exponía en forma de decreto. El informe se publicó posteriormente en el Dziennik Ustaw (Diario de las Leyes).

Se debe señalar que esta competencia se ejercía con total independencia con respecto al control judicial de la constitucionalidad del derecho efectuado por el Tribunal Constitucional. En 1997, la competencia en cuestión desaparece por completo de las prerrogativas del Tribunal Constitucional. Se adujeron dos razones principales para su supresión. En primer lugar, la aplicación de las leyes se hacía demasiado rígida: ¿cómo podía ser válida una sola interpretación de la disposición concreta de una ley en los miles de casos en que debía aplicarse? En segundo lugar, esta competencia parecía interferir con el poder legislativo del Parlamento.

La interpretación de la Constitución hecha por el Tribunal Constitucional presenta algunos rasgos característicos:

- En primer lugar, el Tribunal en el ejercicio de sus competencias actúa siempre y únicamente de conformidad con la petición de los sujetos específicamente autorizados a recurrir al tribunal en virtud de la constitución.

- En segundo lugar, el Tribunal Constitucional aduce muy detenidamente las razones por las que da una interpretación de este tipo y no otra. La parte justificativa de la decisión suele ser bastante voluminosa. Ella puede contener una centena de páginas y la haría parecer como un tratado científico.

- En tercer lugar, al leer las normas de la constitución, el Tribunal Constitucional se refiere a valores (y utiliza estos valores). Por lo tanto, se basa en un determinado sistema axiológico.

Revela así los valores que subyacen a las normas y que influyen en la interpretación dada. Por ejemplo, el Tribunal Constitucional establece una jerarquía de los principios del derecho, dando primacía a un principio del derecho a expensas otro. Así ocurre con la autorización para el sacrificio animal

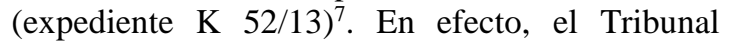
Constitucional ha dado preeminencia a la libertad de culto con respecto a la moral pública (que se opone al sufrimiento animal).

- En cuarto lugar, la interpretación del Tribunal Constitucional está marcada por una cierta "ortodoxia". Con ello entendemos que el Tribunal siempre hace referencia a sus posiciones anteriores, a su autoridad. El Tribunal Constitucional a menudo aplica la interpretación sistémica con referencia a su propia jurisprudencia. Este "bucle" que caracteriza el trabajo de interpretación del Tribunal Constitucional refleja su preocupación por garantizar una estabilidad en la interpretación de las disposiciones constitucionales.

El Tribunal Constitucional tuvo que interpretar la Constitución durante la crisis constitucional de los años 2015 y 2016. En particular, se pronunció sobre los fundamentos jurídicos de su poder de decisión y sobre los mandatos de los magistrados. Para servirme de un ejemplo, "una ley de corrección" del 22 de diciembre de 2015 (era una revisión de una ley sobre el Tribunal Constitucional también de 2015) ha sido por así decirlo "inmunizada" por el Sejm (Cámara baja del Parlamento), contra todo intento de control por parte del Tribunal Constitucional. En efecto, el Sejm decidió hacerla entrar en vigor sin demora (habiendo caso omiso intencionalmente al vacatio legis $^{8}$ ). Esta medida tenía por objeto impedir que el tribunal constitucional procediera al control de la constitucionalidad de esta ley. El legislador quería, pues, extraerlo del ámbito del constitucionalismo.

7 OTK ZU n 11, posición 118.

8 Período que transcurre desde la publicación de una norma hasta su entrada en vigor. 
El razonamiento que el Sejm presentó en esa ocasión fue el siguiente: si el tribunal constitucional había examinado una ley en virtud de la cual debía pronunciarse, no podía en modo alguno emitir su inconstitucionalidad. Sólo era posible una decisión positiva. En consecuencia, el Tribunal Constitucional se basó directamente en las disposiciones constitucionales y en la Ley de 2015 en su versión revisada, pero excluyó algunas de sus disposiciones ${ }^{9}$. Por consiguiente, el Tribunal Constitucional debía interpretar disposiciones específicas de la Constitución de la República de Polonia.

Desde el punto de vista formal, la interpretación dada por el Tribunal Constitucional no está supervisada por otras autoridades, incluidos los tribunales. Sin embargo, el propio Tribunal toma nota de la forma en que una norma jurídica, una vez interpretada, funciona en el sistema jurídico. Y, una vez más, si los tribunales de derecho común siguen planteando al Tribunal Constitucional cuestiones jurídicas relativas a una disposición interpretada, ello demuestra que la interpretación no se ha asimilado bien en la práctica o por alguna razón no cumple bien su función. No se puede excluir que el Tribunal Constitucional se pronuncie una vez más sobre esta cuestión.

Existe poca controversia con respecto a la interpretación de las normas entre el tribunal Constitucional, el Tribunal Europeo de Derechos Humanos y/o los tribunales de la Unión Europea. Por su parte, el tribunal constitucional sigue muy de cerca la jurisprudencia de estos tribunales, entra en diálogo con ellos y se asocia al principio de no afrontarlos. Uno de los métodos del tribunal constitucional es "arreglárselas" y dar una interpretación proeuropea de las disposiciones del Derecho polaco.

\section{CONCLUSIONES}

El nuevo papel de los tribunales constitucionales en el mundo ha venido a cambiar la concepción del poder judicial y en muchos casos el equilibrio de poderes. Para el caso de Polonia el Tribunal Constitucional Polaco se creó en la Carta Política de 1991 como el órgano encargado de controlar y juzgar las normas del derecho. Su función primaria ha redundado en una constitucionalización del derecho.

Esta función de interpretación pro-constitucional se autolimita debido a que no puede llevar a la creación de una norma nueva, ausente previamente del sistema jurídico polaco. Bajo este panorama, la clase política en el debate que abrió hace algún tiempo para acabar con lo que popularmente se denomina la "juezocracia", lo ha hecho con la finalidad de limitar el poder del Tribunal Constitucional Polaco, quien interpreta la Constitución a través de una interpretación derivada. Sin embargo, el interés de crear una Carta Política pura y perfecta, es decir que no requiera interpretaciones se vuelve un reto imposible de materializar que nos llevaría quedarnos en la espera de una utopía pura.

La reflexión desarrollada en el presente artículo nos lleva a concluir que la problemática de la interpretación de la Constitución tiene una importancia vital hoy día en muchas democracias y Polonia no es la excepción. La característica de las disposiciones constitucionales dificulta su lectura textual lo que favorece el trabajo de interpretación y el redescubrimiento. La actividad de la interpretación de la Carta Política es de una importancia vital para su aplicación de forma efectiva, ya que ella permite a las normas adquirir un contenido y alcance particular.

Este escrito también lleva a concluir la existencia y utilidad de los métodos y técnicas de interpretación de la normatividad, actividad que es realizada por excelencia por el juez constitucional. Se citó la interpretación dinámica y la interpretación proconstitucional de las normas jurídicas vigentes, ya mencionada en la parte preliminar como variante de la interpretación dinámica el principio de constitucionalismo. Esto se traduce en decisiones sobre la interpretación y sobre el alcance de la norma superior.

Bajo este panorama, el Juez constitucional polaco debe continuar con su importante labor de defensa de la Constitución, observando los principios de la Carta de 1997 y la jurisprudencia de los Tribunales de la Unión Europea. 


\section{REFERENCIAS BIBLIOGRÁFICAS}

I. Böckenförde, E. W. (2000). Estudios sobre el

Estado de Derecho y la democracia. Madrid, Editorial Trotta.

\section{REFERENCIAS NORMATIVAS}

II. Polonia. Sentencia del Tribunal Constitucional de Polonia, K 47/15 del 9 de marzo de 2016.

III. Polonia. Sentencia del Tribunal Constitucional K 52/13.

IV. Polonia. Sentencia del Tribunal Constitucional de Polonia, SK 29/13, OTK ZU 2015.

V. Polonia. Sentencia del Tribunal Constitucional de Polonia, K 24/15 del 11 de octubre de 2016 OTK ZU 2016A.

VI. Polonia. Sentencia del Tribunal Constitucional de Polonia, K 6/14, de 23 de noviembre de 2016 decisión K 9/11 OTK ZU 2011, n 6A expediente K9/11. 